\title{
Development and validation of a TaqMan RT-PCR method for identification of mayonnaise spoilage yeast Pichia kudriavzevii
}

\author{
M. Y. Syromyatnikov ${ }^{*}$ (D) S. V. Kiryanova ${ }^{2}$ and V. N. Popov ${ }^{1}$
}

\begin{abstract}
Food spoilage and its contamination with yeast and mold is a serious problem of food industry. Despite the high fat content, mayonnaise is an attractive substrate for food spoilage microorganisms. The aim of this study was to develop a method for yeast identification in mayonnaise and to test commercially available mayonnaises for the presence of these contaminating microorganisms. Based on the sequencing of intergenic regions ITS1 and ITS2, we identified a yeast microorganism that causes mayonnaise spoilage. We found that DNA sequences were more than $99 \%$ identical to the GenBank DNA sequences from Pichia kudriavzevii. We developed a specific to P. kudriavzevii TaqMan probe and primers. The reaction conditions were optimized regarding to the components concentration and temperature cycle. The minimum amount of $P$. kudriavzevii DNA that could be detected by developed method was $50 \mathrm{fg}$. The minimal number of $P$. kudriavzevii cells that could be detected by developed method without pre-enrichment was 50. We tested verified method with DNAs from microorganisms of different taxonomic groups that were obtained from three collections of microorganisms. Finally, we analyzed 20 different brands of mayonnaise from 14 producers and 10 different brands of mayonnaise sauce from seven producers. We determined the Cq parameter that characterizes transition of the fluorescence curve to the logarithmic phase and, therefore, correlates with the extent of sample contamination with $P$. kudriavzevii yeast. P. kudriavzevii was detected in six analyzed samples of mayonnaise and one sample of mayonnaise sauce.
\end{abstract}

Keywords: Mayonnaise spoilage, Pichia kudriavzevii, TaqMan, Identification, Mayonnaise screening

\section{Introduction}

Food spoilage and its contamination with pathogenic microorganisms is a serious problem of food industry. Often, contaminating agents are eukaryotic microorganisms, such as yeast and molds. Microbial contamination is very common for the products with a high fat content, e.g., mayonnaises. Mayonnaise is a semi-liquid emulsion of oil, egg yolks, whole eggs, vinegar, and/or lemon juice and some other ingredients (salt, spices, glucose, etc.) with pH 3.6 to 4.6 (Jay et al. 2005). The risk of spoilage is exacerbated by the refusal of food-producing companies to use preservatives. Despite the high fat content,

\footnotetext{
*Correspondence: syromyatnikov@bio.vsu.ru

1 Department of Genetic, Cytology and Bioengineering, Voronezh State University, 1 Universitetskaya pl., Voronezh 394018, Russia

Full list of author information is available at the end of the article
}

mayonnaise is an attractive substrate for food spoilage microorganisms and even some pathogenic microbes. One of the first studies on mayonnaise contamination was performed by Fabian and Wethington (1950), who identified yeast in mayonnaise samples; other authors have demonstrated later that yeast, e.g., Saccharomyces bailii, often contaminates mayonnaise (Kurtzman et al. 1971). The prevalent contaminating bacteria in mayonnaises are halophilic/halotolerant species, such as Micrococcus and Bacillus (Sagdic et al. 2017).

Among potential sources of microbial contamination are eggs, because egg yolk is one of the main mayonnaise ingredients. Bacteria, such as Escherichia coli, Shigella spp. and yeasts, such as Candida albicans (Lee et al. 2017), and Coxiella burnetii (Tatsumi et al. 2006) were found in eggs; for the latter, the identification method using real-time PCR has been developed 
(Sadamasu et al. 2006). In another study, Candida sp. was found in 231 out of 380 mayonnaise samples tested (Musgrove et al. 2008). Other common eukaryotic egg-contaminating microorganisms are molds, mostly from the genera Mucor, Penicillium, Hormodendron, and Cladosporium; Penicillium and Cladosporium being the most frequent causes for fungal spoilage of eggs (Jay et al. 2005). Representatives of other genera, such as Cryptococcus, Hansenula, Hyphopichia, Metschnikowia, Rhodotorula, Sporobolomyces, and Torulaspora have been identified in eggs as well. The most dangerous mayonnaise-contaminating microorganism is Salmonella. In Rio Grande do Sul (Brazil), homemade mayonnaise accounts for $17 \%$ cases of salmonellosis (Capalonga et al. 2014). Changes in pH and temperature can control the growth of Salmonella enteritidis (Keerthirathne et al. 2016), and the model describing the growth of this microorganism in mayonnaise at different temperatures has been proposed (Elias et al. 2016).

Scientists have also been searching for biologically active compounds capable of inhibiting microbial growth in mayonnaise. For example, chitosan was found to suppress the growth of Lactobacillus, but not of the yeast Saccharomyces bailii (Oh et al. 2001).

The common method for identification of microorganisms in mayonnaise is microbial plating on a nutrient medium; however, molecular genetic methods have also gained a wide recognition, because these methods are considerably less time-consuming. Thus, real-time PCR method was suggested for Shigella sp. detection (Villalobo and Torres 1998) and successfully used for Salmonella enterica identification in mayonnaise (Almeida et al. 2013). Another real-time PCR-based system (MicroSEQ ${ }^{\circledR}$ L. monocytogenes Detection Kit) was used for Listeria monocytigenes detection in mayonnaise-containing food products (Tebbs et al. 2011). A method for differentiation of contaminating yeasts have been developed based on the high-resolution melting analysis (Erdem et al. 2016); however, this method did not provide rapid and precise identification of Pichia spp.

The aim of this study was to develop a method for yeast identification in mayonnaise and to test commercially available mayonnaises for the presence of these contaminating microorganisms.

\section{Materials and methods Samples}

Commercially available mayonnaises and mayonnaise sauces of different brands available in Russia market were bought for testing and stored at $+4{ }^{\circ} \mathrm{C}$ in sealed packaging before analysis.

\section{Microbiological analysis}

To test for microbial contamination, mayonnaise samples $(1 \mathrm{ml})$ were diluted two times and plated on the medium containing $40 \mathrm{~g} / \mathrm{l}$ glucose, $10 \mathrm{~g} / \mathrm{l}$ peptone, and $15 \mathrm{~g} / \mathrm{l}$ agar. All medium components were dissolved in hot water, and $\mathrm{pH}$ was adjusted so that $\mathrm{pH}$ after sterilization was 6.5 at $25^{\circ} \mathrm{C}$. Chloramphenicol was added at a final concentration of $50 \mathrm{mg} / \mathrm{l}$.

To enrich yeast cells, mayonnaise samples $(1 \mathrm{ml})$ were enriched by incubating for $19 \mathrm{~h}$ in $30 \mathrm{ml}$ of Sabouraud broth containing $20 \mathrm{~g} / \mathrm{l}$ peptone and $40 \mathrm{~g} / \mathrm{l}$ glucose, and then centrifuged for $5 \mathrm{~min}$ at $10,000 \mathrm{~g}$. The resulting pellet was used for DNA isolation.

\section{Molecular identification of yeasts}

DNA from the grown colonies was isolated using commercially available PROBA-GS kit (DNA Technology, Russia). Polymerase chain reaction was performed with an Eppendorf MasterCycler Personal cycler. Each PCR reaction mixture contained $2.5 \mu$ l of $10 \times$ reaction buffer, $1 \mu \mathrm{l}$ of $10 \mathrm{mM}$ dNTPs, $1 \mu \mathrm{l}$ of $10 \mu \mathrm{M}$ forward primer, $1 \mu \mathrm{l}$ of $10 \mu \mathrm{M}$ reverse primer, $3 \mu \mathrm{l}$ of $25 \mathrm{mM} \mathrm{Mg}^{2+}, 1 \mu \mathrm{g}$ of template DNA, 2.5 units of thermostable Taq DNA polymerase (Evrogen, Russia), and deionized water (up to $25 \mu \mathrm{l}$ ). PCR regime included initial denaturation at $94{ }^{\circ} \mathrm{C}$ for $5 \mathrm{~min} ; 35$ cycles of denaturation at $94{ }^{\circ} \mathrm{C}$ for $30 \mathrm{~s}$, annealing at $54{ }^{\circ} \mathrm{C}$ for $30 \mathrm{~s}$, elongation at $72{ }^{\circ} \mathrm{C}$ for $45 \mathrm{~s}$; final elongation at $72{ }^{\circ} \mathrm{C}$ for $10 \mathrm{~min}$. Fungal-specific primers for molecular identification were: direct ITS1 primer-5'-TCCGTAGGT GAACCTGCGG; reverse ITS4 primer-5'-TCCTCC GCTTATTGATATGC (White et al. 1990).

PCR products were stained with ethidium bromide and visualized at $312 \mathrm{~nm}$ with a TCO-20LM transilluminator after electrophoresis in $2 \%$ agarose gel.

RCR products were purified from the gel with a Cleanup Standard kit (Evrogen, Russia) and sequenced with an Applied Biosystems 3500 automated sequencer using a BigDye Terminator v3.1 Cycle Sequencing Kit and ITS1/ITS4 primers.

\section{Development TaqMan RT-PCR}

Nucleotide sequence alignment was carried out in MEGA6 tool (Tamura et al. 2013). Primer/probe sets for identification of $P$. kudriavzevii were designed according to the following criteria: primer length between 18 and $30 \mathrm{bp}$; no distinctive hairpin structures and dimers; GC content from 20 to $80 \%$; minimal G/C content at the $3^{\prime}$ end of the primers; minimum identical nucleotides together in TaqMan probes; no $G$ at the probe $5^{\prime}$-end; PCR-product size from 80 to $250 \mathrm{bp}$; 
annealing temperature of the probe at least $4{ }^{\circ} \mathrm{C}$ above annealing temperature of the primers.

Real-time PCR was carried out in a Bio-Rad CFX96 Real-time PCR Detection System (Bio-Rad, USA) using commercially available qPCRmix-HS mixture (Eurogen, Russia) according to the following protocol: initial denaturation at $94{ }^{\circ} \mathrm{C}$ for $5 \mathrm{~min}$; 37 cycles of denaturation at $94{ }^{\circ} \mathrm{C}$ for $30 \mathrm{~s}$, annealing at $54{ }^{\circ} \mathrm{C}$ for $30 \mathrm{~s}$, elongation at $72{ }^{\circ} \mathrm{C}$ for $30 \mathrm{~s}$.

\section{Results}

\section{Bioinformatics analysis and primer/probe design}

To identify contaminating yeast species, we selected eight commercially available mayonnaise samples and plated them on agar plates. After 3-day growth, yeast-resembling colonies were isolated for further molecular biological studies. Yeast presence was found in four samples of mayonnaise. After DNA isolation we sequenced the DNA fragments that contained 5.8S rRNA gene and two internally transcribed spacers, ITS1 and ITS2 and compared them to nucleotide sequences from the GenBank database. We found out that all sequences were more than 99\% identical to the DNA sequences from P. kudriavzevii. The sequenced fragments were also analyzed using the data from the Boldsystem (http://www.boldsystems.org) that confirmed that the identified microorganism was P. kudriavzevii. The obtained sequences were deposited in the Genbank database under the corresponding numbers: MH626402.1, MH626403.1, MH626404.1, MH626405.1, MH626406.1, MH626407.1, MH626408.1 and MH626409.1.
We analyzed over 180 registered in GenBank DNA sequences of $5.8 \mathrm{~S}, 18 \mathrm{~S}$ and $28 \mathrm{~S}$ ribosomal RNA genes and internally transcribed spacers ITS1 and ITS2 for $P$. kudriavzevii and synonymic species (Issatchenkia orientalis, Candida krusei) to identify specific sequences. The primer/probe sets for identification of $P$. kudriavzevii were suggested (Table 1).

More rigorous analysis of the designed primer/probe sets revealed possible heterodimer ( 8 nucleotides) formation between the TaqMan probe and direct primer and between the TaqMan probe and reverse primer in the primer/probe sets 4 and 6 , respectively, so they were omitted from further study. The remaining four primer/ probe sets were synthesized and verified in real-time PCR with yeast DNA. No TaqMan probe hybridization was observed for the sets 1 and 3; out of sets 2 and 4, TaqMan probe from set 2 hybridized more strongly and at earlier PCR cycles, so this primer/probe set was used in further studies. Specificity to $P$. kudriavzevii DNA mostly provides TaqMan probe and a reverse primer from set 2 . The site for TaqMan probe hybridization is specific for this yeast. Homologous sites of the TaqMan probe hybridization to DNA of the closest eukaryotic microorganisms registered in the GenBank are shown in Fig. 1.

The site for attaching the TaqMan probe to DNA was highly specific to this species of yeast. There was no variability of this site inside the P. kudriavzevii species. However, it was found that there is still a small probability of a positive TaqMan reaction with DNA from yeasts that phylogenetically most close to Pichia sp.-Martiniozyma sp. and Saturnispora sp. (Fig. 2).

Table 1 Suggested primers and TaqMan probes for identification of $P$. kudriavzevii

\begin{tabular}{|c|c|c|}
\hline Primer/probe set & Nucleotide sequences & $\begin{array}{l}\text { PCR product } \\
\text { length, b.p. }\end{array}$ \\
\hline 1 & $\begin{array}{l}\text { Forward primer GAATTGCAGCCATCGTGAATC } \\
\text { TaqMan probe FAM-TCGTTTCCATCTTGCGCGTGC-BHQ1 } \\
\text { Reverse primer CTCCGACGCTCTTTACACG }\end{array}$ & 149 \\
\hline $2^{a}$ & $\begin{array}{l}\text { Forward primer GCGAAATGCGATACCTAGTG } \\
\text { TaqMan probe FAM-TGCAGCCATCGTGAATCATCGAGT-BHQ1 } \\
\text { Reverse primer GATGGAAACGACGCTCAAA }\end{array}$ & 114 \\
\hline 3 & $\begin{array}{l}\text { Forward primer GCGGACGACGTGTAAAG } \\
\text { TaqMan Probe FAM-GAGCGAGTGTTGCGAGACAACAA-BHQ1 } \\
\text { Reverse primer CGGGTATTCCTACCTGATTTG }\end{array}$ & 156 \\
\hline 4 & $\begin{array}{l}\text { Forward primer GAGCGTCGTTTCCATCTT } \\
\text { TaqMan probe FAM-CAGCTCCGACGCTCTTTACACGTC-BHQ1 } \\
\text { Reverse primer CAGCTTCGCTCCCTTTC }\end{array}$ & 102 \\
\hline 5 & $\begin{array}{l}\text { Forward primer GATCTCTTGGTTCTCGCATC } \\
\text { TaqMan probe FAM-CACACTAGGTATCGCATTTCGCTGC-BHQ1 } \\
\text { Reverse primer GCGTTCAAGAACTCGATGA }\end{array}$ & 92 \\
\hline 6 & $\begin{array}{l}\text { Forward primer GCAGCCATCGTGAATCAT } \\
\text { TaqMan probe FAM-TTTGAGCGTCGTTTCCATCTTGCG-BHQ1 } \\
\text { Reverse primer AGCTCCGACGCTCTTTA }\end{array}$ & 146 \\
\hline
\end{tabular}

\footnotetext{
a The most optimal primer/probe sets for identification of $P$. kudriavzevii
} 


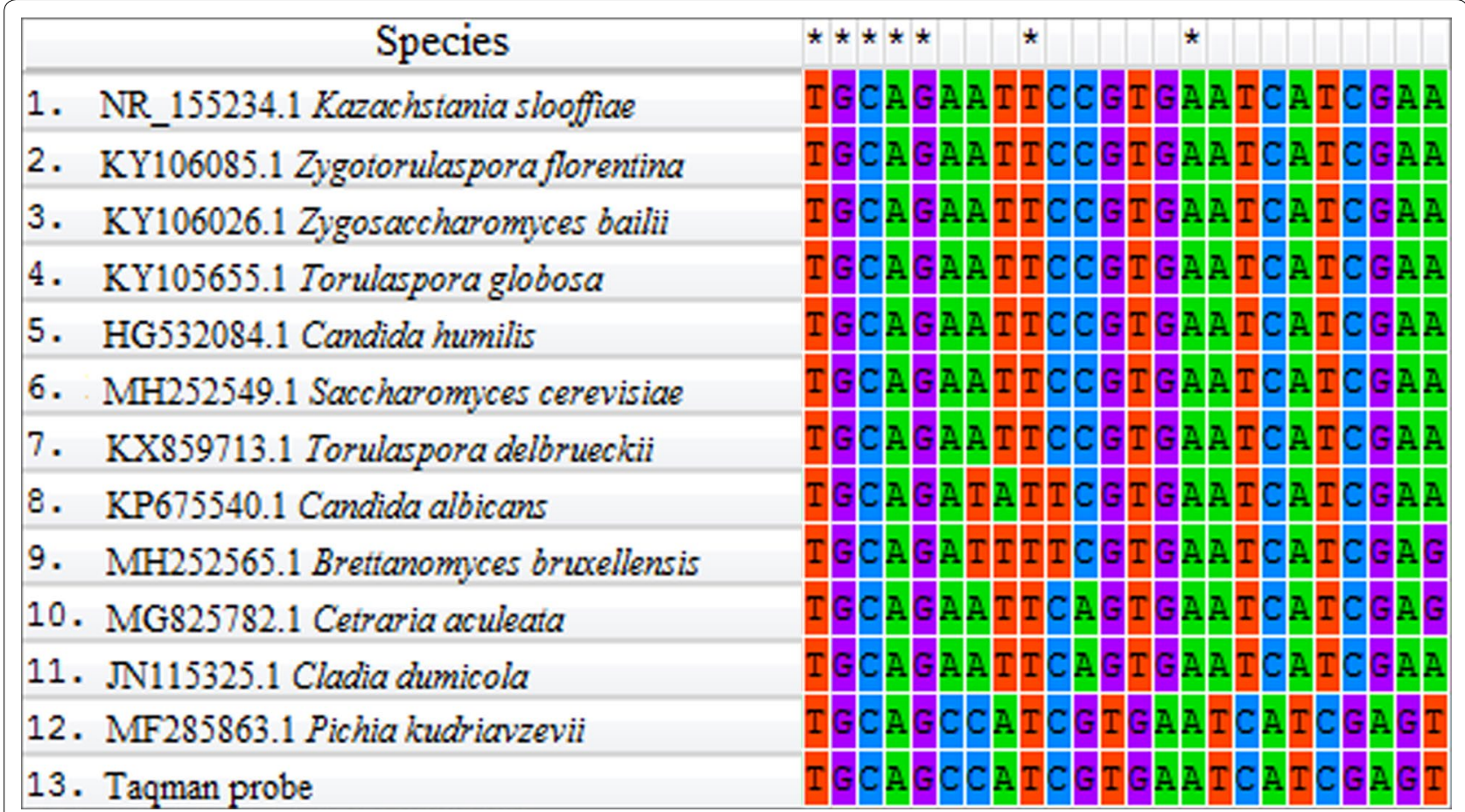

Fig. 1 Homologous sequences of the P. kudriavzevii DNA and DNA of other closest eukaryotic microorganisms

Based on the PCR product expected size and the primer annealing temperature, the PCR regime used was as follows: $94{ }^{\circ} \mathrm{C}$ for $4 \mathrm{~min}$ followed by 37 cycles of $94{ }^{\circ} \mathrm{C}$ for $30 \mathrm{~s}, 50-58{ }^{\circ} \mathrm{C}$ for $30 \mathrm{~s}, 72{ }^{\circ} \mathrm{C}$ for $30 \mathrm{~s}$. We ran the reaction at five different primer annealing temperatures: $50,52,54,56$, and $58{ }^{\circ} \mathrm{C}$. At all the temperatures tested, the fluorescence curve reached the logarithmic phase at the same cycle number, although the total levels of fluorescence were higher at 50,52 , and $54{ }^{\circ} \mathrm{C}$. Since higher primer annealing temperatures increase primer specificity, $54{ }^{\circ} \mathrm{C}$ was chosen as the optimal primer annealing temperature in our experiments. We also tested the effect of TaqMan probe concentration (100, 200, and $500 \mathrm{nM}$ ) on the reaction parameters and found that amplification using $200 \mathrm{nM}$ TaqMan probe produced more stable and pronounced amplification curves; the total level of fluorescence with $200 \mathrm{nM}$ TaqMan probe was higher than when using $100 \mathrm{nM}$ TaqMan probe. The optimal number of cycles was 37, because in such a number of cycles fluorescence was not observed in samples without $P$. kudriavzevii DNA.

\section{Estimation of method sensitivity}

DNA was isolated from $P$. kudriavzevii culture and diluted to $1 \mathrm{ng} / \mu \mathrm{l}$, diluted 1: 10, 1: 100, 1:1000, and 1: 10,000 , and used as a template in real-time PCR with the developed primer/probe set $(1 \mu \mathrm{l}$ per reaction tube), see
Fig. 3. The linear dependence of the Cq parameter on the concentration of the genomic DNA of $P$. kudriavzevii was observed (Fig. 4), which means that the reaction is specific only to the target DNA without the formation of non-specific products.

The minimum amount of Pichia sp. DNA that could be detected by our method was $50 \mathrm{fg}$. To estimate the minimal number of live cells sufficient for $P$. kudriavzevii detection, total DNA was isolated from 10, 50, 100, 1000, and 10,000 cells of P. kudriavzevii and used as a template in the same PCR. The minimal number of yeast that could be detected by our method without pre-enrichment was 50 cells.

\section{Verification of the specificity of the developed primer/ probe set for Pichia kudriavzevii}

To verify the specificity of the developed primer/probe, we tested it in PCR with DNAs from microorganisms of different taxonomic groups that were obtained from the Russian Collection of Microorganisms (VKM) at the Skryabin Institute of Microbial Biochemistry and Physiology (Pushchino, Russia), State collection of microorganisms that cause dangerous, particularly dangerous, including zooanthroponotic animals diseases (VKOM) at the Federal Research Center for Virology and Microbiology (Pokrov, Russia) and Russian collection of industrial microorganisms (VKMP) State Research Institute 


\begin{tabular}{ll} 
Martiniozyma asiatica & GCAGCGAAATGCGATAATTAATGTGA \\
Martiniozyma abiesophila & GCAGCGAAATGCGATAATTAATGTGA \\
Saturnispora zaruensis & GCAGCGAAATGCGATAACTAATGTGA \\
Saturnispora mendoncae & GCAGCGAAATGCGATAACTAATGTGA \\
Saturnispora diversa & GCAGCGAAATGCGATAACTAATGTGA \\
\begin{tabular}{|l} 
Forward primer \\
\hline
\end{tabular} & -- GCGAAATGCGATACCTAGTG--- \\
\hline
\end{tabular}

Saturnispora zaruensis

Saturnispora mendoncae

Saturnispora diversa

Martiniozyma asiatica

Martiniozyma abiesophila

Reverse primer

\section{CTGTTTGAGCGTCAGTTCATTCTAT \\ CTGTTTGAGCGTCGGTTCAATCTGA \\ CTGTTTGAGCGTCGGTTCAATCTTT \\ TTGTTTGAGCGTCGTTTCCCTCTGT \\ TTGTTTGAGCGTCGTTTCCTTCTGT \\ - - - TTTGAGCGTCGTTTCCATC - - -}

Saturnispora zaruensis

Saturnispora mendoncae

Saturnispora diversa

Martiniozyma asiatica

Martiniozyma abiesophila

Taqman
AATTGCAGGCATCGTGAATCATCGAGTTCT

AATTGCAGGCATCGTGAATCATCGAGTTCT

AATTGCAGGCATCGTGAATCATCGAGTTCT

AATTGCAGGCATCGTGAATCATCGAGTTCT

AATTGCAGGCATCGTGAATCATCGAGTTCT

- - - TGCAGCCATCGTGAATCATCGAGT - - -

$* * * * * \quad * * * * * * * * * * * * * * * * * *$

Fig. 2 Sites for primers and TaqMan probe hybridization of P. kudriavzevii DNA and Martiniozyma sp. and Saturnispora sp. DNA

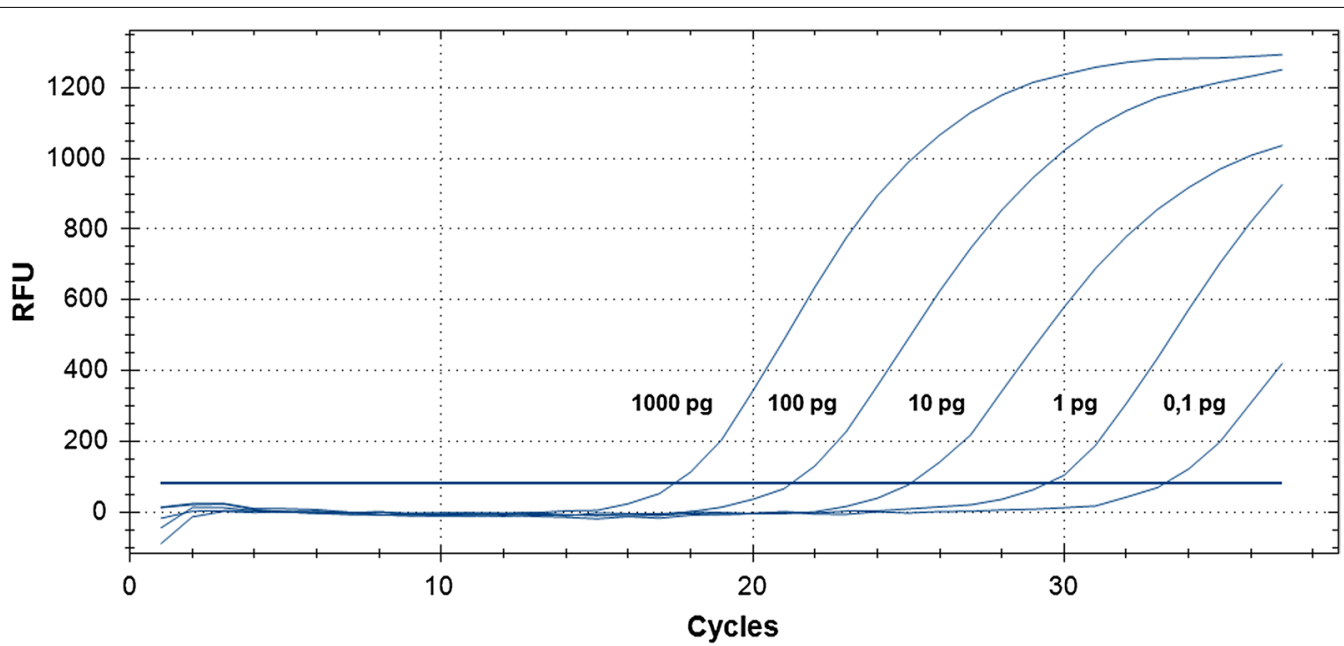

Fig. 3 The influence of different amount of P. kudriavzevii genomic DNA per reaction and Cq parameter

of Genetics and Selection of Industrial Microorganisms (Moscow, Russia) (Table 2).

PCR proceeded only with microorganisms from the Pichia genus. No increase in fluorescence was observed for other yeast, molds and bacteria. We also contaminated 23 samples of preliminary tested microbe-free mayonnaise with P. kudriavzevii. After these samples were enriched, DNA was isolated and used in the 


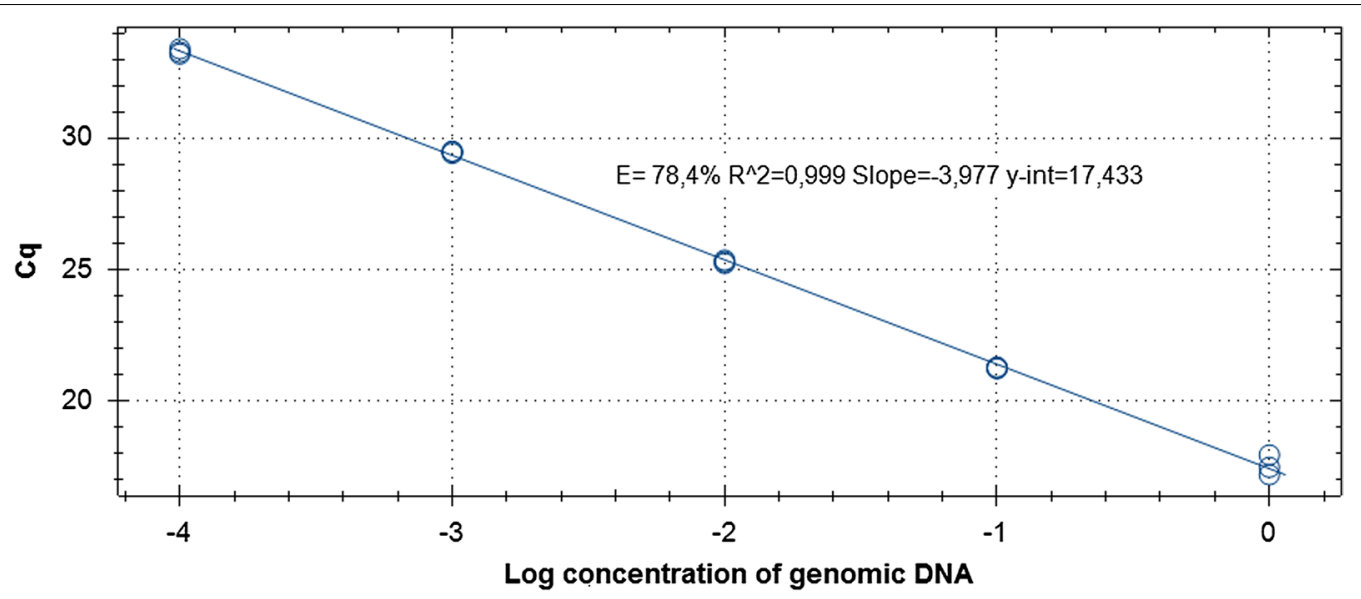

Fig. 4 The dependence of Cq parameter on concentration of genomic DNA of P. kudriavzevii

real-time PCR with the developed primer/probe set. In all 23 cases, the reaction was positive, i.e., the reproducibility of the method was $100 \%$.

\section{Testing commercially available mayonnaises} and mayonnaise sauces for microbial contamination

We analyzed 20 different brands of mayonnaise from 14 producers and 10 samples of mayonnaise sauce from seven different brands. We determined the $\mathrm{Cq}$ parameter that characterizes transition of the fluorescence curve to the logarithmic phase and, therefore, correlates with the extent of sample contamination with P. kudriavzevii yeast.

The increase in fluorescence was observed in six analyzed samples of mayonnaise: sample $1(\mathrm{Cq}=20,13)$, sample $13(\mathrm{Cq}=31,70)$, sample $14(\mathrm{Cq}=28,61)$, sample $15(\mathrm{Cq}=30,40)$, sample $17(\mathrm{Cq}=33,95)$ and sample $17(\mathrm{Cq}=34,50)$. 1. The increase in fluorescence was observed in sample 1 of mayonnaise sauce $(\mathrm{Cq}=34,86)$. The Cq values for the "positive" samples differed, thereby indicating different extents of contamination with $P$. kudriavzevii.

\section{Discussion}

Detection and quantitative estimation of yeast and mold contamination is a sanitary requirement for most food products, although contaminating microorganisms are not usually identified to genera or species. Different yeast and molds might develop differently in different food products. The yeast $P$. kudriavzevii, that were identified by us in mayonnaise samples, tolerate high fat content and low $\mathrm{pH}$ and were detected in Chinese vinegar ( $\mathrm{Li}$ et al. 2014). P. fermentas were found in freshly cut apples (Graça et al. 2015); P. anomala were identified in maize silage that it characterized by low $\mathrm{pH}$ values (Santos et al.
2017). Pichia yeast has been also found in other products: P. guilliermondii (Sangorrin et al. 2013), P. membranifaciens, and $P$. manshurica contaminate wines; $P$. fermentans and P. kudriavzevii were found in cheeses (Gonçalves Dos Santos et al. 2017). Also, yeasts of these taxonomic groups were identified in products with high fat content (Arroyo-Lopez et al. 2012). Therefore, early detection of $P$. kudriavzevii yeast will ensure removal of contaminated products from realization and eliminate the use of infected ingredients in the production of mayonnaise and mayonnaise sauces.

In this study, we developed a primer/probe set to a region of Pichia genome that included internally transcribed spacer ITS1, 5.8S rRNA gene and internally transcribed spacer ITS2. This region was chosen because the information on its nucleotide sequence in many organisms (including Pichia yeast) is widely available in sequence databases, such as GenBank. In addition, the genomic DNA of eukaryotic microorganisms contains this region in several copies. This increases the sensitivity of the method. Using this information, we were able to identify taxon-characteristic conserved nucleotide sequences and to develop a corresponding probe/primer set for $P$. kudriavzevii identification. The primer/probe set used in this study was highly specific and did not anneal to the DNA from other than Pichia microorganisms. The sensitivity of our method without pre-enrichment was 50 yeast cells.

We have found that primers and the TaqMan probe can react with DNA of other species from the Pichia genus. Given the purpose for which the identification system was developed (detection of mayonnaise spoilage yeast), this fact does not have any negative impact. For the same reason, the hypothetical interaction of the developed TaqMan probe with DNA of Martiniozyma sp. 
Table 2 Verification of the specificity of the developed method with microorganisms from different taxonomic groups

\begin{tabular}{|c|c|c|c|}
\hline Organism & Collection & $\begin{array}{l}\text { Collection } \\
\text { number } \\
\text { or strain }\end{array}$ & $\begin{array}{l}\text { Reaction } \\
\text { with TaqMan }\end{array}$ \\
\hline
\end{tabular}

Mold and yeast

Alternaria alternata

Aspergillus flavus

Aspergillus carbonarius

Chaetomium globosum

Fusarium verticillioides

Geotrichum candidum

Penicillium funiculosum

Penicillium pinophilum

Phanerochaete chrys-

osporium

Phlebia albida

Pleurotus ostreatus

Trichoderma reesei

Trichoderma viride

Brettanomyces custersianus

Candida pseudotropicalis

Candida tropicalis

Dekkera anomala

Pichia fermentans

Pichia kudriavzevii

Pichia membranifaciens

Saccharomyces cerevisiae

Yarrowia lipolytica

Zygosaccharomyces rouxii

Bacteria

Bacillus subtilis

Cellulomonas flavigena

Corynebacterium glutamicum

Lactobacillus plantarum

Pseudomonas fluorescens

Streptomyces fulvoviridis

Listeria monocytogenes

Staphylococcus aureus

Pseudomonas aeruginosa

Bacillus cereus

Escherichia coli

Salmonella enteritidis

Listeria ivanovii

Micrococcus luteus

Rhodococcus equi

and Saturnispora sp. also will not have a negative effect, because these yeasts are very close to Pichia sp. (Kurtzman et al. 2008; Kurtzman 2015). We couldn't check experimentally whether there is a TaqMan probe interaction with DNA of these microorganisms because we did not have verified collection strains of the Martiniozyma sp. and Saturnispora sp.

The developed method for rapid identification of Pichia yeast using real-time PCR reduces the time required for analysis from 5 days to $24 \mathrm{~h}$, which allows rapid detection of contamination source and timed removal of the contaminated produce.

Molecular genetic identification of microorganisms in food products with high fat content (such as mayonnaise) is often impeded by the difficulties in the DNA isolation. In the procedure developed by us, yeast cells were concentrated by centrifugation after preliminary enrichment of the culture. By doing this, we solved both methodical problems, i.e., we increased the method sensitivity by enriching the microorganism culture and removed fats that interfered with DNA isolation.

The developed procedure is rapid and simple and could be carried out in any laboratory equipped with a realtime PCR system. It could also be used for testing other food products.

Screening of commercially available mayonnaises and mayonnaise sauces demonstrated that 6 out of 20 mayonnaises and 1 out of 10 mayonnaise sauces were contaminated with Pichia yeast.

As follows from the high Cq value, one of the mayonnaise samples displayed an extremely high abundance of Pichia sp. cells; two other samples were moderately contaminated, and the rest of the samples exhibited low extent of yeast contamination.

Note that the positive count in mayonnaises was higher than in mayonnaise sauces due, probably, to the fact that mayonnaise sauces contain preservatives, while mayonnaises are often positioned as "organic" products free of chemical additives, such as preservatives or aromatic compounds.

In conclusion, we identified yeasts that able to contaminate mayonnaise. Based on the sequencing of intergenic regions ITS1 and ITS2, we shown that spoilage mayonnaise yeasts are $P$. kudriavzevii. We developed a specific to $P$. kudriavzevii TaqMan probe and primers. The reaction was optimized for the components concentration and temperature cycle. The minimal number of yeast cells that could be detected by our method without preenrichment was 50 cells. Screening of commercially available mayonnaises and mayonnaise sauces demonstrated that 6 out of 20 mayonnaises and 1 out of 10 mayonnaise sauces were contaminated with Pichia yeast. The developed procedure is rapid and simple and could be carried out in any laboratory equipped with a real-time PCR system. 


\section{Abbreviations}

DNA: deoxyribonucleic acid; RNA: ribonucleic acid; PCR: polymerase chain reaction; ITS: internal transcribed spacer.

\section{Authors' contributions}

MS carried out molecular genetic studies and wrote the manuscript; SK performed microbiological and molecular genetics experiments; VN analyzed the data. All authors read and approved the final manuscript.

\section{Author details}

${ }^{1}$ Department of Genetic, Cytology and Bioengineering, Voronezh State University, 1 Universitetskaya pl., Voronezh 394018, Russia. ${ }^{2}$ Innovative Center "Biruch-NT", EFKO Group of Companies, Malybykovo, 1 Belaya Vezha Str., Belgorod Region 309927, Russia.

\section{Acknowledgements}

We thank Anastasia Kokina and Olga Savinkova for their experimental assistance. We also thank EFKO Group of Companies for provision of materials for analysis.

\section{Competing interests}

The authors declare that they have no competing interests.

\section{Availability of data and materials}

The datasets supporting the conclusions of this article are included within the article.

\section{Consent for publication}

Not applicable.

\section{Ethics approval and consent to participate}

This article does not contain any studies with human participants or animals performed by any of the authors.

\section{Funding}

This study was supported by Ministry of Education and Science of the Russian Federation (Agreement N 14.577.21.0257, unique agreement code RFMEFI57717X0257).

\section{Publisher's Note}

Springer Nature remains neutral with regard to jurisdictional claims in published maps and institutional affiliations.

Received: 7 July 2018 Accepted: 17 November 2018

Published online: 22 November 2018

\section{References}

Almeida C, Cerqueira L, Azevedo NF, Vieira MJ (2013) Detection of Salmonella enterica serovar enteritidis using real time PCR, immunocapture assay, PNA FISH and standard culture methods in different types of food samples. Int J Food Microbiol 161(1):16-22. https://doi. org/10.1016/j.ijfoodmicro.2012.11.014

Arroyo-Lopez FN, Romero-Gil V, Bautista-Gallego J, Rodriguez-Gomez F, Jimenez-Diaz R, Garcia-Garcia P, Querol A, Garrido-Fernandez A (2012) Yeasts in table olive processing: desirable or spoilage microorganisms? Int J Food Microbiol 160(1):42-49. https://doi.org/10.1016/j.ijfoodmicr 0.2012 .08 .003

Capalonga R, Ramos RC, Both JM, Soeiro ML, Longaray SM, Haas S, Tondo EC (2014) Salmonella serotypes, resistance patterns, and food vehicles of salmonellosis in southern Brazil between 2007 and 2012. J Infect Dev Ctries 8(7):811-817. https://doi.org/10.3855/jidc.3791

Elias SO, Alvarenga VO, Longhi DA, Sant'Ana ADS, Tondo EC (2016) Modeling growth kinetic parameters of Salmonella enteritidis SE86 on homemade mayonnaise under isothermal and nonisothermal conditions. Foodborne Pathog Dis 13(8):462-467. https://doi.org/10.1089/fpd.2015.204
Erdem M, Kesmen Z, Özbekar E, Çetin B, Yetim H (2016) Application of highresolution melting analysis for differentiation of spoilage yeasts. J Microbiol 54(9):618-625. https://doi.org/10.1007/s12275-016-6017-8

Fabian FW, Wethington MO (1950) Bacterial and chemical analyses of mayonnaise, salad dressing, and related products. J Food Sci 15(2):138-145. https://doi.org/10.1111/j.1365-2621.1950.tb16460.x

Gonçalves Dos Santos MTP, Benito MJ, Cordoba MG, Alvarenga N, de Herrera SRMS (2017) Yeast community in traditional Portuguese Serpa cheese by culture-dependent and -independent DNA approaches. Int J Food Microbiol 262:63-70. https://doi.org/10.1016/j.ijfoodmicro.2017.09.013

Graça A, Santo D, Esteves E, Nunes C, Abadias M, Quintas C (2015) Evaluation of microbial quality and yeast diversity in fresh-cut apple. Food Microbiol 51:179-185. https://doi.org/10.1016/j.fm.2015.06.003

Jay JM, Loessner MJ, Golden DA (2005) Modern food microbiology, 7th edn. Springer, New York

Keerthirathne TP, Ross K, Fallowfield H, Whiley H (2016) A review of temperature, $\mathrm{pH}$, and other factors that influence the survival of Salmonella in mayonnaise and other raw egg products. Pathogens 5(4):63. https://doi. org/10.3390/pathogens5040063

Kurtzman CP (2015) Description of Martiniozyma gen. nov. and transfer of seven Candida species to Saturnispora as new combinations. Antonie Van Leeuwenhoek 108(4):803-809. https://doi.org/10.1007/s1048 2-015-0536-x

Kurtzman CP, Rogers R, Hesseltine CW (1971) Microbiological spoilage of mayonnaise and salad dressings. Appl Microbiol 21(5):870-874

Kurtzman CP, Robnett CJ, Basehoar-Powers E (2008) Phylogenetic relationships among species of Pichia, Issatchenkia and Williopsis determined from multigene sequence analysis, and the proposal of Barnettozyma gen. nov., Lindnera gen. nov. and Wickerhamomyces gen. nov. FEMS Yeast Res 8(6):939-954. https://doi.org/10.1111/j.1567-1364.2008.00419.x

Lee SI, Lee H, Jablonski PG, Choe JC, Husby M (2017) Microbial abundance on the eggs of a passerine bird and related fitness consequences between urban and rural habitats. PLoS ONE 12(9):e0185411. https://doi. org/10.1371/journal.pone.0185411

Li P, Li S, Cheng L, Luo L (2014) Analyzing the relation between the microbial diversity of DaQu and the turbidity spoilage of traditional Chinese vinegar. Appl Microbiol Biotechnol 98(13):6073-6084. https://doi. org/10.1007/s00253-014-5697-4

Musgrove MT, Jones DR, Hinton A Jr, Ingram KD, Northcutt JK (2008) Identification of yeasts isolated from commercial shell eggs stored at refrigerated temperatures. J Food Prot 71(6):1258-1261. https://doi. org/10.4315/0362-028X-71.6.1258

Oh HI, Kim YJ, Chang EJ, Kim JY (2001) Antimicrobial characteristics of chitosans against food spoilage microorganisms in liquid media and mayonnaise. Biosci Biotechnol Biochem 65(11):2378-2383. https://doi. org/10.1271/bbb.65.2378

Sadamasu K, Tabei Y, Shinkai T, Hasegawa M, Kaneko S, Hirai A, Nakama A, Ishizaki N, Odagiri M, Kamata S, Yano K, Kai A, Morozumi S (2006) Development of effective detection method for Coxiella burnetii in mayonnaise by real-time PCR and investigation of C. burnetii contamination in commercial mayonnaise in Tokyo. Shokuhin Eiseigaku Zasshi 7(1):1-8. https:// doi.org/10.3358/shokueishi.47.1

Sagdic O, Tornuk F, Karasu S, Durak MZ, Arici M (2017) Microbial ecology of mayonnaise, margarine, and sauces. Quant Microbiol Food Process Model Microb Ecol. https://doi.org/10.1002/9781118823071.ch26

Sangorrin MP, Garcia V, Lopes CA, Saez JS, Martinez C, Gang MA (2013) Molecular and physiological comparison of spoilage wine yeasts. J Appl Microbiol 14(4):1066-1074. https://doi.org/10.1111/jam.12134

Santos MC, Golt C, Joerger RD, Mechor GD, Mourão GB, Kung L Jr (2017) Identification of the major yeasts isolated from high moisture corn and corn silages in the United States using genetic and biochemical methods. J Dairy Sci 100(2):1151-1160. https://doi.org/10.3168/jds.2016-11450

Tamura K, Stecher G, Peterson D, Filipski A, Kumar S (2013) MEGA6: molecular evolutionary genetics analysis version 6.0. Mol Biol Evol 30:2725-2729. https://doi.org/10.1093/molbev/mst197

Tatsumi N, Baumgartner A, Qiao Y, Yamamoto I, Yamaguchi K (2006) Detection of Coxiella burnetii in market chicken eggs and mayonnaise. Ann NY Acad Sci 1078:502-505. https://doi.org/10.1196/annals.1374.096 
Tebbs RS, Balachandran P, Wong LY, Zoder P, Furtado MR, Petrauskene OV, Cao $Y$ (2011) Evaluation of applied biosystems MicroSeq real-time PCR system for detection of Listeria monocytogenes in food. Performance tested method 011002. J AOAC Int 94(5):1481-1489

Villalobo E, Torres A (1998) PCR for detection of Shigella spp. in mayonnaise. Appl Environ Microbiol 64(4):1242-1245
White TJ, Bruns T, Lee S, Taylor J (1990) Amplification and direct sequencing of fungal ribosomal RNA genes for phylogenetics. In: Innis MA, Gelfand DH, Sninsky JJ, White TJ (eds) PCR protocols: a guide to methods and applications. Academic Press, New York, pp 315-322

\section{Submit your manuscript to a SpringerOpen ${ }^{\circ}$ journal and benefit from:}

- Convenient online submission

- Rigorous peer review

- Open access: articles freely available online

- High visibility within the field

- Retaining the copyright to your article

Submit your next manuscript at $\boldsymbol{\nabla}$ springeropen.com 